\title{
Entrenamiento de fuerza con bandas elásticas en niños y adolescentes: una revisión sistemática \\ Resistance training using elastic band in children and adolescents. A systematic review
}

\author{
Belén Lagares Vázquez, José Antonio Rebollo
}

Universidad de Huelva (España)

\begin{abstract}
Resumen. El objetivo de este estudio fue realizar una revisión sistemática para conocer el estado actual de los efectos del entrenamiento de fuerza a partir de dispositivos elásticos en niños y adolescentes. La búsqueda se realizó en las bases de datos Web of Science y Pubmed. De los 38 resultados iniciales, se seleccionaron 8 artículos que cumplían los criterios de elegibilidad establecidos con anterioridad. Para realizar esta revisión sistemática se empleó la declaración PRISMA. Los resultados mostraron mejoras en la fuerza muscular y en diversas variables de salud y rendimiento. El entrenamiento con estos dispositivos obtuvo mejoras en la capacidad de sprint, salto vertical, cambio de dirección y sentadilla. Además, también se hallaron mejoras en la composición corporal, en el equilibrio, en la prevención de lesiones y una mayor adherencia al programa de ejercicio físico. En la actualidad existe una disminución de la fuerza muscular en población infantil y adolescente a nivel mundial. Esta situación puede derivar en problemas adversos para la salud. El entrenamiento de fuerza con bandas elástica se presenta como una herramienta útil y eficaz tanto en el contexto escolar como deportivo. No obstante, son necesarios más estudios que muestren los beneficios del entrenamiento de fuerza a partir de este recurso con el fin de establecer estrategias para mejorar la salud y calidad de vida en esta población.
\end{abstract}

Palabras claves: bandas elásticas; entrenamiento; niños y adolescentes.

\begin{abstract}
The aim of this study was to carry out a systematic review to determine the current status of the effects of resistance training using elastic devices in children and adolescents. The search was performed in the Web of Science and Pubmed databases. Of the 38 initial results, 8 articles were selected that met the previously established eligibility criteria. The PRISMA statement was used to conduct this systematic review. The results showed improvements in muscle strength and in various health and performance variables. Training with these devices obtained improvements in sprint, vertical jump, change of direction and squat. In addition, improvements in body composition, balance, injury prevention and increased adherence to the physical exercise programme were also found. There is currently a worldwide decline in muscle strength in children and adolescents. This situation can lead to adverse health problems. Strength training with elastic bands is presented as a useful and effective tool in both school and sports contexts. However, more studies are needed to show the benefits of strength training from this resource in order to establish strategies to improve health and quality of life in this population.
\end{abstract}

Key words: elastic bands; training; children and adolescents.

\section{Introducción}

El entrenamiento de fuerza en edades tempranas está considerablemente recomendado por las organizaciones relevantes en los campos de la medicina del deporte, la ciencia de la actividad física y la pediatría. Existe un posicionamiento internacional que refuerza y afianza la recomendación y promoción de programas de acondicionamiento neuromuscular durante la niñez y la adolescencia, siempre y cuando estén apropiadamente supervisados y diseñados por personas cualificadas (Lloyd, et al., 2014). Las nuevas recomendaciones de la Orga-

Fecha recepción: 14-05-21. Fecha de aceptación: 19-10-21

Jose Antonio Rebollo

joseantonio.rebollo@dempc.uhu.es nización Mundial de la Salud (OMS) establecen que niños y adolescentes deben hacer actividades de fuerza al menos tres veces a la semana (Bull, et al., 2020).

La variable de fuerza muscular en niñas, niños y adolescentes a lo largo de los años muestra una tendencia descendente. En un estudio, donde se incluyen más de 9 millones de niños y niñas entre 9 y 17 años, evaluaron la fuerza muscular del tronco entre los años 1964 y 2017 y se muestra que esta variable aumenta de manera débil hasta el año 2010 y a partir de éste, disminuye de una manera radical (Kaster, et al., 2020). Otro estudio nacional de tendencia, incluyó diferentes test de evaluación de la condición física, especialmente de fuerza, en los que se evidenció que entre los años 2001 y 2006 se produjo un descenso acentuado de la fuerza muscular, 
tanto en miembros superiores como inferiores, en adolescentes españoles (Moliner-Urdiales, et al., 2010).

Esta disminución se evidencia a nivel mundial. De manera más concreta, niños y niñas con niveles insuficientes de fuerza muscular pueden carecer de un requisito importante para desarrollar habilidades de movimiento fundamentales. En consecuencia, sufren de una menor competencia que conduce a una baja confianza en el movimiento y posteriormente puede causar un comportamiento sedentario. Este déficit de fuerza muscular puede actuar como un desencadenante que conduce a resultados adversos para la salud (Chaabene, et al., 2020).

Además, en niños y adolescentes, el entrenamiento de fuerza tiene efectos positivos sobre multitud de aspectos, entre ellos se encuentran la mejora de habilidades motrices básicas, composición corporal, rendimiento escolar, capacidades cognitivas y descanso nocturno (Collins, et al., 2019; Collins, et al., 2018; Kovacevic, et al., 2018). Cuanto antes se incorpore, mayores serán las mejoras en el sistema motor y estarán más aptos para las actividades físicas o deportivas (Pochetti, et al., 2018).

Aunque existe una gran variedad de ejercicios para mejorar la aptitud muscular, es importante seleccionar ejercicios apropiados al nivel de condición física y experiencia previa. Para niñas, niños y adolescentes se han empleado máquinas, pesas libres, bandas elásticas, balones medicinales y ejercicios con el propio peso corporal. En algunos casos puede ser apropiado comenzar con movimientos multiarticulares, centrándonos en mejorar patrones de movimiento fundamentales (Faigenbaum, et al., 2009).

Las bandas elásticas son uno de los equipos más utilizados para el entrenamiento de fuerza ya que pueden ser utilizadas por personas de todas las edades. Su dureza varía según los colores y se puede aplicar eficazmente en movimientos de una o varias articulaciones (Özsu, 2018). Estos elementos elásticos han sido frecuentemente utilizados en rehabilitación aunque resultan interesantes para conseguir adaptaciones efectivas en cualquier persona ya que proporcionan una sobrecarga progresiva de manera segura (Aloui, et al., 2018).

La ganancia de fuerza es similar con el uso de dispositivos elásticos en comparación con dispositivos convencionales. Por lo que se apuesta por el uso de este material de bajo coste, facilidad de manejo y que se puede utilizar en diferentes lugares (Santos, et al., 2019). En adultos mayores también se evidencia mejoras significativas a partir del uso de bandas elásticas en agili- dad y equilibrio dinámico (Miranda-Aguilar, et al., 2020).

También en mujeres posmenopáusicas un año de entrenamiento de resistencia progresivo con bandas elásticas tiene efectos beneficiosos antiinflamatorios reduciendo el dolor musculoesquelético (Gómez, et al., 2018). El entrenamiento con resistencia elástica mejora la fuerza muscular y la capacidad funcional a lo largo de la vida. Además, parece ser eficaz para mejorar la composición corporal y obtener resultados positivos en algunos biomarcadores de salud, aunque es necesario seguir investigando. (Colado, et al., 2020).

En relación a lo citado anteriormente, se plantea la necesidad de incorporar programas de ejercicio físico donde el eje central sea el desarrollo de la fuerza muscular para abordar todas las esferas que conforman la salud del niño (Chulvi-Medrano, et al., 2018). Por otro lado, se manifiesta la necesidad de intervenir en ámbito escolar, destacando la importancia de la asignatura de Educación física (López, et al., 2021).

La importancia del entrenamiento de fuerza en población infanto-juvenil resulta evidente a lo largo de la literatura científica. Debido a la disminución de esta capacidad actualmente, y a la escasez de propuestas eficaces a través de estos elementos, los objetivos de esta revisión sistemática fueron identificar y examinar los programas que investigaron los efectos positivos de las intervenciones sobre el desarrollo de la fuerza a partir de bandas elásticas en niños y adolescentes.

\section{Método}

En este estudio llevo a cabo esta revisión sistemática en la que se empleó la declaración PRISMA y la guía práctica de revisiones sistemáticas, incluyendo las principales secciones, con o sin meta-análisis (Moher, et al., 2015; Urrútia \& Bonfill, 2010).

\section{Criterios de elegibilidad}

Los criterios de inclusión aplicados a este estudio fueron: a) manuscritos a texto completo, b) fecha de publicación de los trabajos entre 2010 y 2021, c) idioma: inglés o español, d) que tuvieran grupo control y experimental, e) que fuese una intervención, estudio experimental o quasi-experimental, o que se tratara de un ensayo controlado aleatorio. Por tanto, los artículos fueron incluidos a partir de los diferentes criterios de elegibilidad planteados.

\section{Fuentes de información}

La búsqueda de los artículos mostrados fue realizada 
en dos bases de datos (Web Of Science y Pubmed) desde noviembre de 2020 hasta marzo de 2021. Para la realización de la misma se establecieron dos bloques de búsqueda diferenciados: 1) elastic band training AND 2) children OR adolescents.

\section{Selección de estudios y proceso de extracción de datos}

Una vez introducidos los criterios de elegibilidad, se analizaron el título y el abstract de todos los artículos encontrados con el fin de localizar aquellos de mayor relevancia y excluir aquellos que no cumplían los criterios de inclusión. De esta manera se seleccionaron un total de ocho artículos que fueron utilizados para la extracción de información tomando la temática «entrenamiento con bandas elásticas» como principal cuestión a tener en cuenta en las diferentes investigaciones.

Con el propósito de aminorar el sesgo de selección, cada estudio fue revisado de forma independiente por dos investigadores, quienes decidieron si un documento se ajustaba o no a los criterios de inclusión.

\section{Evaluación de calidad}

La evaluación de calidad de los documentos seleccionados se realizó usando la evaluación estándar Qualsyst para estudios cuantitativos (Kmet, et al., 2004). Este análisis incluyó 14 ítems relacionados con aspectos como el diseño de la investigación, la muestra, la metodología, el análisis de datos, los resultados y las conclusiones. Cada criterio fue puntuado con 2 (satisfactorio), 1 (parcialmente satisfactorio) y 0 (no satisfactorio), y NA (no aplicable). La puntuación final fue obtenida a raíz de la siguiente fórmula [(«número de satisfactorio» x 2) + («parcialmente satisfactorio» x 1) / 28 - (no aplicables $\mathrm{x} 2)]$.

\section{Resultados}

\section{Selección de estudios}

Tras la realización de la búsqueda inicial se obtuvieron 33 resultados. Los documentos fueron analizados y otros 5 estudios adicionales fueron identificados en las referencias de estos o por otras fuentes. A continuación, se descartaron aquellos artículos duplicados, lo que supuso la exclusión de 6 estudios. De los restantes, 21 fueron retirados por no corresponder a la temática y 3 después de aplicar los criterios de exclusión. Finalmente, tras la realización del análisis, 8 estudios fueron incluidos en esta revisión (Figura 1).

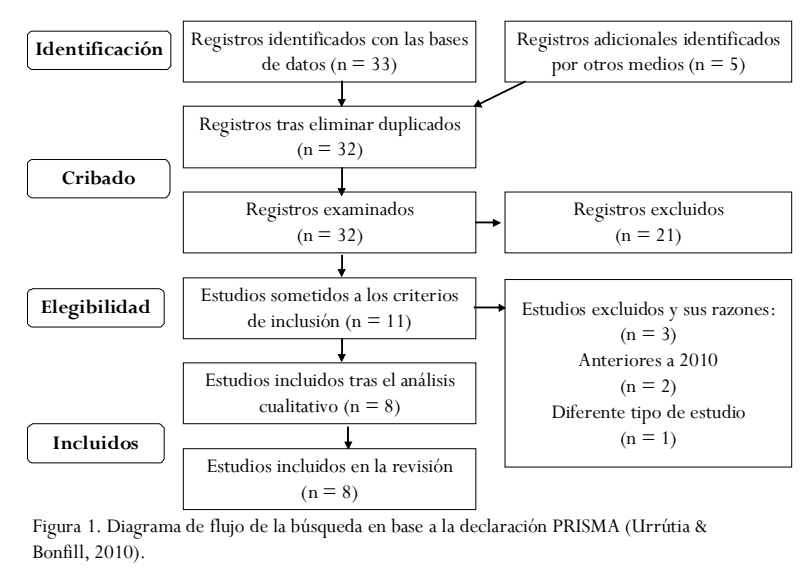

\section{Calidad de los estudios}

En el acuerdo entre los evaluadores se obtuvo un punto de corte liberal $(>0,55)$. Los puntajes generales asignados por el primer observador variaron entre 0,64 y 0,93 y los del segundo de 0,61 a 0,93 (ver tabla 1).

\begin{tabular}{|c|c|c|}
\hline $\begin{array}{l}\text { Estudios } \\
\end{array}$ & Observador 1 & Observador 2 \\
\hline Lubans et al. (2010) & 0,93 & 0,93 \\
\hline Coskun \& Sahin (2014) & 0,64 & 0,61 \\
\hline Batalha et al. (2015) & 0,93 & 0,89 \\
\hline Kordi et al. (2016) & 0,89 & 0,89 \\
\hline Sahin et al. (2016) & 0,68 & 0,68 \\
\hline Özsu (2018) & 0,82 & 0,79 \\
\hline Aloui et al. (2018) & 0,93 & 0,93 \\
\hline Hammami et al. (2021) & 0,96 & 0,96 \\
\hline
\end{tabular}

\section{Características de los estudios}

Las características principales de los estudios seleccionados se presentan a continuación (Tabla 2).

Tal como muestra la tabla 2, se analizaron un total de ocho artículos sobre intervenciones que usan elementos de resistencia elástica en niños o adolescentes. Las edades de los participantes se diferencian en dos grandes grupos. Los estudios realizados en niños abarcan edades comprendidas entre 7 y 12 años (Coskun \& Sahin, 2014; Kordi, et al., 2016; Özsu, 2018; ahin, et al., 2016). Las edades de los sujetos adolescentes se encuentra entre 14 y 19 años (Aloui, et al., 2018; Batalha, et al., 2015; Hammami, et al., 2021; Lubans, et al., 2010).

En cuanto a los objetivos de los estudios, varios de ellos evaluaron los efectos entre dos programas de fuerza en el que uno de ellos se empleó resistencias elásticas (Coskun \& Sahin, 2014; Lubans, et al., 2010; ahin, et al., 2016). El resto de las investigaciones examinaron los efectos que producían los entrenamientos que utilizaban este tipo de elementos. En cinco de los artículos analizados, existe una tendencia orientada a la salud a partir de las intervenciones realizadas (Batalha, et al., 2015; Coskun \& Sahin, 2014; Kordi, et al., 2016; Lubans, et al., 2010; Özsu, 2018). Tres de ellos se centraron en 
Tabla 2.

\begin{tabular}{|c|c|c|c|c|c|}
\hline \multirow[t]{2}{*}{ Estudios } & \multirow{2}{*}{$\begin{array}{l}\text { Participantes (n) } \\
\text { Edad (años) Sexo }\end{array}$} & \multirow[t]{2}{*}{ Objetivo del estudio } & \multicolumn{2}{|l|}{ Programa de intervención } & \multirow[t]{2}{*}{ Principales resultados } \\
\hline & & & Grupo control & Grupo experimental & \\
\hline $\begin{array}{l}\text { Lubans et } \\
\text { al. (2010) }\end{array}$ & $\begin{array}{l}\text { Grupo peso libre: } 37 \\
\text { Grupo tubos elásticos: } 41 \\
\text { Grupo control: } 30 \\
\text { Edad: } 15 \pm 0.7 \\
\text { Chicas y chicos }\end{array}$ & $\begin{array}{l}\text { Evaluar la eficacia y viabilidad de dos } \\
\text { programas de entrenamiento de fuerza } \\
\text { realizados en contexto escolar para } \\
\text { mejorar la aptitud muscular y la } \\
\text { composición corporal en adolescentes }\end{array}$ & $\begin{array}{l}\text { No participó en ningún tipo de } \\
\text { entrenamiento }\end{array}$ & $\begin{array}{l}\text { Programa de entrenamiento de fuerza } \\
\text { durante } 8 \text { semanas, } 2 \text { días/semana } \\
\text { usando tubos elásticos o peso libre } \\
\text { Volumen: } 2 \times 8-12 \text { repeticiones } \\
\text { 60-90" descanso entre series } \\
\text { (10 ejercicios) } \\
\text { Intensidad: escala de Borg }(15-18)\end{array}$ & $\begin{array}{l}\text { Tanto chicos como chicas completaron } 8 \\
\text { semanas de entrenamiento y mejoraron } \\
\text { significativamente su composición corporal y } \\
\text { fuerza muscular en comparación con el grupo } \\
\text { control. La adherencia al programa fue mayor } \\
\text { entre los participantes con tubos elásticos. }\end{array}$ \\
\hline $\begin{array}{l}\text { Coskun \& } \\
\text { Sahin } \\
(2014)\end{array}$ & $\begin{array}{l}\text { Grupo experimental: } 16 \\
\text { Grupo control: } 14 \\
\text { Edad no especificada } \\
\text { Chicas y chicos }\end{array}$ & $\begin{array}{l}\text { Investigar los efectos de diferentes } \\
\text { entrenamientos de fuerza en un período } \\
\text { entrenado y un período no entrenado en } \\
\text { niños }\end{array}$ & $\begin{array}{l}\text { Entrenamiento con su propio } \\
\text { peso corporal } 2 \text { veces por semana } \\
\text { Volumen: } 1-2 \text { series y } 10-12 \\
\text { repeticiones ( } 7 \text { ejercicios) } \\
\text { Intensidad: establecida con } 10 \\
\text { RM } \\
\end{array}$ & $\begin{array}{l}\text { Ejercicios con bandas elásticas } \\
\text { (amarillas, verdes y azules) } 2 \\
\text { días/semana durante } 6 \text { semanas } \\
\text { Volumen: } 1-2 \text { series y } 10-12 \\
\text { repeticiones ( } 7 \text { ejercicios) } \\
\text { Intensidad: establecida con } 10 \mathrm{RM}\end{array}$ & $\begin{array}{l}\text { En el período de desentrenamiento, se } \\
\text { determinó menor disminución en la fuerza en } \\
\text { el grupo que entrenó con bandas elásticas } \\
\text { comparado con el que entrenó con el propio } \\
\text { peso. }\end{array}$ \\
\hline $\begin{array}{l}\text { talha et } \\
\text { (2015) }\end{array}$ & $\begin{array}{l}\text { Grupo experimental: } 20 \\
(14.65 \pm 0.49) \\
\text { Grupo entrenamiento: } 20 \\
(14.45 \pm 0.51) \\
\text { Grupo control: } 16(14.69 \\
\pm 0.48) \\
\text { Solo chicos }\end{array}$ & $\begin{array}{l}\text { Evaluar los efectos de un programa de } \\
\text { entrenamiento de fuerza compensatorio } \\
\text { en la fuerza y la estabilidad de los } \\
\text { músculos rotadores del hombro de } \\
\text { jóvenes nadadores de competición }\end{array}$ & $\begin{array}{l}\text { No participó en ningún tipo de } \\
\text { entrenamiento }\end{array}$ & $\begin{array}{l}16 \text { semanas } \\
\text { Grupo entrenamiento: solo } \\
\text { entrenamiento acuático } \\
\text { Grupo experimental: entrenamiento } \\
\text { acuático }+ \text { entrenamiento con bandas } \\
\text { elásticas (Thera-Band) } 3 \text { veces/semana } \\
\text { Volumen: } 3 \times 20-30 \text { repeticiones ( } 3 \\
\text { ejercicios) } \\
\text { Intensidad: hasta agotamiento }\end{array}$ & $\begin{array}{l}\text { Un entrenamiento de fuerza compensatorio } \\
\text { tiene efectos beneficiosos en los músculos del } \\
\text { manguito rotador del hombro }\end{array}$ \\
\hline $\begin{array}{l}\text { Kordi et } \\
\text { al. (2016) }\end{array}$ & $\begin{array}{l}\text { Grupo experimental: } 15 \\
(8.01 \pm 0.54) \\
\text { Grupo control: } 15(7.70 \pm \\
0.63) \\
\text { Chicas y chicos }\end{array}$ & $\begin{array}{l}\text { Evaluar los efectos del entrenamiento de } \\
\text { fuerza con bandas elásticas para mejorar } \\
\text { el equilibrio estático y dinámico en niños } \\
\text { con trastornos de desarrollo de la } \\
\text { coordinación }\end{array}$ & $\begin{array}{l}\text { Clases de educación física } \\
\text { Ningún entrenamiento de fuerza }\end{array}$ & $\begin{array}{l}\text { Entrenamiento de fuerza (12 semanas, } \\
2 \text { sesiones/semana) } \\
\text { Volumen: } 3 \times 10-15 \text { repeticiones ( } 6 \\
\text { ejercicios con bandas elásticas } \\
\text { theraband) }\end{array}$ & $\begin{array}{l}\text { El entrenamiento de fuerza llevó a una mejora } \\
\text { en el equilibrio estático pero no hubo una } \\
\text { mejora en el equilibrio dinámico. }\end{array}$ \\
\hline $\begin{array}{l}\text { hin et } \\
(2016)\end{array}$ & $\begin{array}{l}\text { Grupo 1: } 8(11.1 \pm 0.83) \\
\text { Grupo 2: } 8(11.5 \pm 0.53) \\
\text { Grupo control: } 5(11.8 \pm \\
0.45) \\
\text { Sexo no especificado }\end{array}$ & $\begin{array}{l}\text { Examinar el efecto a corto plazo de los } \\
\text { ejercicios de sentadilla realizados con } \\
\text { una banda elástica sobre la sentadilla } \\
\text { estática y dinámica y los saltos verticales } \\
\text { en preadolescentes }\end{array}$ & $\begin{array}{l}\text { No participó en ningún tipo de } \\
\text { entrenamiento }\end{array}$ & $\begin{array}{l}\text { Programa de sentadilla durante } 6 \\
\text { semanas, } 2 \text { días/semana } \\
\text { Volumen: } 2 \times 20-30 \text { repeticiones } \\
\text { Grupo 1: con bandas elásticas (azules y } \\
\text { amarillas) } \\
\text { Grupo 2: solo con el propio peso } \\
\text { corporal }\end{array}$ & $\begin{array}{l}\text { Se encontraron mayores mejoras en las pruebas } \\
\text { realizadas (salto vertical, sentadilla dinámica y } \\
\text { sentadilla estática) a partir del ejercicio de } \\
\text { sentadillas con bandas elásticas. }\end{array}$ \\
\hline $\begin{array}{l}\ddot{O}_{\text {zsu }} \\
(2018)\end{array}$ & $\begin{array}{l}\text { Grupo experimental: } 50 \\
(8.6 \pm 0.5) \\
\text { Grupo control: } 52(8.6 \pm \\
0.6) \\
\text { Sexo no especificado }\end{array}$ & $\begin{array}{l}\text { Investigar los efectos de los ejercicios } \\
\text { realizados durante } 6 \text { semanas con una } \\
\text { banda de resistencia elástica sobre el } \\
\text { rendimiento funcional (equilibrio, } \\
\text { agilidad, fuerza de agarre manual y } \\
\text { flexibilidad) en niños de } 8 \text { a } 9 \text { años. }\end{array}$ & $\begin{array}{l}\text { No participó en ningún tipo de } \\
\text { actividad física. }\end{array}$ & $\begin{array}{l}\text { Ejercicios usando bandas elásticas } \\
\text { (amarillas) } 3 \text { días/semana, } 45 \\
\text { min/sesión (durante } 6 \text { semanas) } \\
\text { Volumen: } 2 \times 15 \text { repeticiones (10 } \\
\text { ejercicios) }\end{array}$ & $\begin{array}{l}\text { Se determinó que los ejercicios de } 6 \text { semanas } \\
\text { realizados con bandas elásticas aumentaron } \\
\text { significativamente la fuerza de agarre en mano, } \\
\text { sin embargo, no hubo un efecto significativo en } \\
\text { las habilidades de equilibrio, agilidad y } \\
\text { flexibilidad. }\end{array}$ \\
\hline $\begin{array}{l}\text { Aloui et al. } \\
\text { (2018) }\end{array}$ & $\begin{array}{l}\text { Grupo experimental: } 15 \\
(18.3 \pm 0.8) \\
\text { Grupo control: } 15(18.8 \pm \\
0.8) \\
\text { Solo chicos }\end{array}$ & $\begin{array}{l}\text { Evaluar los efectos en las habilidades } \\
\text { relacionadas con el rendimiento de los } \\
\text { jugadores de balonmano masculino } \\
\text { junior cuando una parte de la temporada } \\
\text { fue sustituida por el entrenamiento } \\
\text { basado en bandas elásticas. }\end{array}$ & $\begin{array}{l}\text { Mantuvo su régimen estándar } \\
\text { durante la temporada }\end{array}$ & $\begin{array}{l}\text { Dos ejercicios: extensión de rodilla y } \\
\text { extensión de cadera con bandas } \\
\text { elásticas (theraband: negra, plata y } \\
\text { oro) } 2 \text { sesiones/semana ( } 8 \text { semanas) } \\
\text { Volumen: } 3 x 12-15 \\
\text { Intensidad: esfuerzo máximo }\end{array}$ & $\begin{array}{l}\text { Se mejoró la capacidad muscular, el sprint y la } \\
\text { capacidad de hacer cambios de dirección en los } \\
\text { jugadores adolescentes de élite de balonmano a } \\
\text { partir del entrenamiento con banda elásticas en } \\
\text { relación con el régimen estándar. }\end{array}$ \\
\hline $\begin{array}{l}\text { Hammami } \\
\text { et al. } \\
(2021)\end{array}$ & $\begin{array}{l}\text { Grupo experimental: } 14 \\
(14.86 \pm 0.52) \\
\text { Grupo control: } 13(14.74 \\
\pm 0.36) \\
\text { Solo chicos }\end{array}$ & $\begin{array}{l}\text { Evaluar los cambios en la fuerza } \\
\text { dinámica, reactiva y de potencia y el } \\
\text { equilibrio en jugadores de voleibol } \\
\text { púberes cuando una parte de su } \\
\text { entrenamiento fue reemplazada por un } \\
\text { entrenamiento con bandas elásticas. }\end{array}$ & $\begin{array}{l}\text { Mantuvo su entrenamiento } \\
\text { estándar durante la temporada } \\
\text { régimen y no siguió ninguna } \\
\text { intervención durante este } \\
\text { período }\end{array}$ & $\begin{array}{l}\text { Entrenamiento de fuerza en miembros } \\
\text { inferiores con bandas elásticas (negra, } \\
\text { plata, oro), } 2 \text { sesiones/semana, } 45 \\
\text { min/sesión ( } 8 \text { semanas) } \\
\text { Volumen: } 2 \times 10-12 \text { (semana } 1 \text { y } 2 \text { ) } \\
3 \times 10-12 \text { (semana } 3 \text { y 4) } \\
4 \times 10-15 \text { (semana } 5,6,7 \text { y } 8 \text { ) } \\
\text { Intensidad: RPE (4-6) }\end{array}$ & $\begin{array}{l}\text { Mejora del rendimiento (potencia y fuerza } \\
\text { dinámica, y equilibrio anteroposterior) en } \\
\text { jugadores de voleibol adolescentes. La fuerza } \\
\text { reactiva y otros parámetros relacionados con el } \\
\text { equilibrio no cambiaron con el entrenamiento } \\
\text { de bandas elásticas. }\end{array}$ \\
\hline
\end{tabular}

evaluar el rendimiento de varias variables (Aloui, et al., 2018; Hammami, et al., 2021; ${ }^{a}$ ahin, et al., 2016).

Respecto al programa de intervención, la mayoría de ellos muestran un volumen entre 2-3 series y 10-15 repeticiones con dos sesiones a la semana. En cambio, la intensidad no aparece de manera consensuada a lo largo de las investigaciones. En cuanto al uso de los elementos de resistencia elástica, en cuatro de ellos (Aloui, et al., 2018; Batalha, et al., 2015; Hammami, et al., 2021; Kordi, et al, 2016) se indica que usaron Thera-Band. Además, se nombró el color de las bandas elásticas empleadas por los participantes en los distintos estudios (Aloui, et al., 2018; Coskun \& Sahin, 2014; Hammami, et al., 2021; Özsu, 2018; ${ }^{a}$ ahin, et al., 2016).

Como principales resultados, todos mostraron resultados positivos en cuanto al uso de bandas elásticas. Los estudios que analizaron distintos programas de fuerza, evidenciaron mayores mejoras en la fuerza muscu- lar, en pruebas de salto y sentadilla, e incluso se mostró una mayor adherencia al programa de entrenamiento (Coskun \& Sahin, 2014; Hammami, et al., 2021; Lubans, et al., 2010; a ahin, et al., 2016). Asimismo, también se encontraron efectos beneficiosos en la musculatura, mejoras en el equilibrio estático, aumento en los niveles de fuerza manual y mejores resultados en pruebas de velocidad (Batalha, et al., 2015; Hammami, et al., 2021; Kordi, et al. 2016; Lubans, et al., 2010; Özsu, 2018).

\section{Discusión}

Los objetivos de esta revisión sistemática fueron identificar y examinar los programas que investigaron los efectos de las intervenciones sobre el desarrollo de la fuerza a partir de bandas elásticas en niños y adolescentes. 
Los contextos de los estudios seleccionados se enfocan tanto en el ámbito escolar como fuera de éste. Solo una de las investigaciones se realizó en el ámbito escolar (Lubans, et al., 2010) a diferencia del resto que pertenecían a otros ambientes deportivos, de rendimiento o salud (Aloui, et al., 2018; Batalha, etal., 2015; Coskun \& Sahin, 2014; Hammami, et al., 2021; Kordi, et al., 2016; Özsu, 2018; ${ }^{a}$ ahin, et al., 2016).

Existieron varios estudios en los que participaron tanto chicos como chicas (Coskun \& Sahin, 2014; Kordi, et al., 2016; Lubans, et al., 2010), menos en tres de ellos en los cuales los participantes pertenecían al sexo masculino (Aloui, et al., 2018; Batalha, et al., 2015; Hammami, et al., 2021). Respecto al número de participantes a lo largo de todos los estudios se observó una gran variedad. Se encontraron estudios en los que el número de participantes por grupo fueron menores a veinte (Aloui, et al., 2018; Coskun \& Sahin, 2014; Hammami, et al., 2021; Kordi, et al., 2016; ahin, et al., 2016) a diferencia de aquellos estudios que intervinieron un mínimo de cincuenta personas en cada uno de los grupos (Özsu, 2018).

Correspondiente a los distintos objetivos, de manera general se midió la fuerza muscular en todos los estudios. Varios de los estudios se centraron en medir variables basadas en el rendimiento de los partícipes como el sprint, saltos y cambios de dirección (Aloui, et al., 2018; Hammami, et al., 2021; ahin, et al., 2016). El resto se centró en observar las mejoras relacionadas con la salud como la composición corporal o el equilibrio entre otras (Batalha, et al., 2015; Kordi, et al., 2016; Lubans, et al., 2010).

Respecto al tiempo de las intervenciones, se encontraron tres estudios que emplearon 6 semanas (Coskun \& Sahin, 2014; Özsu, 2018; ahin, et al., 2016), tres realizaron 8 semanas (Aloui, et al., 2018; Hammami, et al., 2021; Lubans, et al., 2010), una de las intervenciones fueron de 12 semanas (Kordi, et al., 2016) y 16 semanas fue el mayor periodo de tiempo encontrado en las investigaciones (Batalha, et al., 2015).

Por otro lado, predominó la ejecución de dos sesiones a la semana en la mayoría de estudios, excepto en dos de los artículos examinados (Batalha, et al., 2015; Özsu, 2018), cuya frecuencia fue de 3 sesiones en una misma semana.

Otras de las variables a tener en cuenta en las investigaciones fue el volumen. La cantidad de ejercicios fue distinta en todos los estudios. Sin embargo, en cuanto a la dosis, se coincidió en aplicar 2 series en cuatro de ellos (Coskun \& Sahin, 2014; Lubans, et al., 2010; Özsu,
2018; a ahin, et al., 2016). Otra opción que presentaron el resto de artículos fue la realización de 3 series (Aloui, et al., 2018; Batalha, et al., 2015; Kordi, et al., 2016). Sin embargo, en uno de ellos se mostró una progresión de 2 a 4 series a lo largo de las semanas (Hammami, et al., 2021).

En cambio, las repeticiones se encontraban entre 8 y 15, menos en uno de los estudios (ahin, et al., 2016) que aumentaron hasta un total de 30 repeticiones. Por otro lado, una de las limitaciones en las variables del entrenamiento, se halla en la intensidad del ejercicio, la cual no apareció consensuada. Solo tres de ellos la nombraron, empleando la escala de Borg (Lubans, et al., 2010), estableciendo la 10 RM (Coskun \& Sahin, 2014) o RPE (Hammami, et al., 2021).

En cuanto a los principales resultados examinados, el entrenamiento de fuerza usando dispositivos elásticos obtuvo diversos beneficios tanto en el aumento del rendimiento (Aloui, et al., 2018; Batalha, et al., 2015; Hammami, et al., 2021) como en la mejora de la salud (Kordi, et al., 2016; Lubans, et al., 2010). Aunque en uno de ellos no se mostraron mejoras significativas en habilidades de equilibrio, agilidad y flexibilidad (Özsu, 2018).Y otro estudio no presentó cambios positivos en otras variables como la fuerza reactiva y otros parámetros relacionados con el equilibrio a partir del entrenamiento con bandas elásticas (Hammami, et al., 2021).

A partir de los estudios analizados, podemos observar la utilidad de los elementos elásticos en estas poblaciones como elemento principal de estudio. Por otro lado, existen varias investigaciones en esta población, que se ayudan de este recurso en sus intervenciones combinándolo con otros tipos de herramientas para obtener beneficios en distintas variables de estudio (Guillot, et al., 2019; Riel, et al., 2016; Terraza-Rebollo, et al., 2017).

Comparando nuestros resultados con otras revisiones sistemáticas, se afirma la efectividad del entrenamiento de fuerza para la mejora de la condición física en niños y niñas con sobrepeso y obesidad (Le-Cerf, et al., 2022). Otro punto de vista ofrecido, se refleja en jóvenes futbolistas, donde además de incluir entrenamiento pliométrico, es necesario otro tipo de entrenamiento de fuerza para generar adaptaciones neuromusculares (Martínez-Pérez \& Vaquero-Cristóbal, 2021). Estos hallazgos junto a los de nuestro estudio, nos llevan a ver la importancia y necesidad de incluir este tipo de intervenciones en nuestra población.

Una de las limitaciones de la revisión realizada se 
encuentra en las diferencias en la muestra, la edad, las variables medidas y la planificación de los entrenamientos, lo cual dificulta la comparación entre los mismos. Por tanto, los resultados encontrados son heterogéneos. En este sentido, futuras investigaciones son necesarias para concretar los efectos producidos a partir del uso de dispositivos elásticos. Además, se debería poner el foco en aspectos que permitieran avanzar en el control y dosificación del entrenamiento empleando este recurso ya que pueden suponer una opción para aquellos profesionales del entrenamiento de fuerza.

Por último, apoyando los resultados de nuestro estudio, el entrenamiento de fuerza con resistencia elástica permite obtener mejoras en la fuerza muscular y en la capacidad funcional a lo largo de la vida (Colado, et al., 2020). Esta revisión sistemática puede tener como principal aplicación práctica servir de utilidad tanto a entrenadores como a docentes a la hora de trabajar la capacidad de la fuerza y conseguir diferentes beneficios usando bandas elásticas en esta población.

\section{Conclusiones}

A partir de este estudio, se concluye que la utilización de elementos elásticos para el trabajo de fuerza en niños y adolescentes puede resultar una herramienta eficaz tanto en la mejora de la salud como en el aumento del rendimiento deportivo. La aplicación del trabajo de fuerza evidencia múltiples beneficios en esta población empleando este tipo de materiales.

Además, las bandas elásticas suponen un recurso útil, económico y llamativo que nos permite realizar una buena planificación en el entrenamiento y educar en la mejora de la calidad de vida. Por lo tanto, se sugiere un mayor uso e inclusión de este recurso en edades tempranas.

\section{Referencias}

Aloui, G., Hammami, M., Fathloun, M., Hermassi, S., Gaamouri, N., Shephard, R. J., \& Chelly, M. S. (2018). Effects of an 8-week in-season elastic band training program on explosive muscle performance, change of direction, and repeated changes of direction in the lower limbs of junior male handball players. The Journal of Strength \& Conditioning Research, 33(7), 1804-1815. DOI: https: / / doi.org/10.1519/JSC.0000000000002786.

Batalha, N., Raimundo, A., Tomas-Carus, P., Paulo, J., Simão, R., \& Silva,A. J. (2015). Does a land-based compensatory strength-training programme influences the rotator cuff balance of young competitive swimmers? European journal of sport science, 15(8), 764-772. DOI: https://doi.org/
10.1080/17461391.2015.1051132.

Bull, F. C., Al-Ansari, S. S., Biddle, S., Borodulin, K., Buman, M. P., Cardon, G., Carty, C., Chaput, J., Chastin, S., Chou, R., Dempsey, P. C., DiPietro, L., Ekelund, U., Firth, J., Friedenreich, C., García, L., Gichu, M., Jago, R., Katzmarzyk, P.T., ... Willumsen, J. F. (2020).World Health Organization 2020 guidelines on physical activity and sedentary behaviour. British journal of sports medicine, 54(24), 1451-1462. DOI: https://doi.org/ 10.1136/bjsports-2020-102955.

Chaabene, H., Lesinski, M., Behm, D. G., \& Granacher, U. (2020). Performance-and health-related benefits of youth resistance training. Sports Orthopaedics and Traumatology. DOI: https://doi.org/10.1016/j.orthtr.2020.05.001.

Colado, J. C., Mena, R., Calatayud, J., Gargallo, P., Flández, J., \& Page, P. (2020). Effects of strength training with variable elastic resistance across the lifespan: a systematic review. Cultura, Ciencia y Deporte, 15(44), 147-164. DOI: https://doi.org/10.12800/ccd.v15i44.1458.

Collins, H., Booth, J. N., Duncan, A., \& Fawkner, S. (2019). The effect of resistance training interventions on fundamental movement skills in youth: a meta-analysis. Sports medicine-open, 5(1), 17. DOI: https://doi.org/10.1186/ s40798-019-0188-x.

Collins, H., Fawkner, S., Booth, J. N., \& Duncan, A. (2018). The effect of resistance training interventions on weight status in youth: a meta-analysis. Sports medicine-open, 4(1), 41. DOI: https://doi.org/10.1186/s40798-018-0154$\mathrm{z}$.

Coskun,A., \& Sahin, G. (2014). Two different strength training and untrained period effects in children. Journal of Physical Education and Sport, 14(1), 42. DOI: https://doi.org/ 10.7752/jpes.2014.01007.

Faigenbaum, A. D., Kraemer,W. J., Blimkie, C. J., Jeffreys, I., Micheli, L. J., Nitka, M., \& Rowland, T.W. (2009).Youth resistance training: updated position statement paper from the national strength and conditioning association. The Journal of Strength \& Conditioning Research, 23, S60-S79. DOI: https://doi.org/10.1519/ JSC.0b013e31819df407.

Gómez, C., Gil, J. M., Carrasco, J. J., Alakhdar,Y., \& ChulviMedrano, I. (2018). Efectos de un año de entrenamiento con bandas elásticas sobre el dolor en mujeres menopáusicas. Fisioterapia, 40(4), 178-182. DOI: https: / /doi.org/10.1016/j.ft.2018.03.003

Guillot, A., Kerautret,Y., Queyrel, F., Schobb,W., \& Di Rienzo, F. (2019). Foam Rolling and Joint Distraction with Elastic Band Training Performed for 5-7 Weeks Respectively Improve Lower Limb Flexibility. Journal of sports science \& medicine, 18(1), 160.

Hammami, R., Gene-Morales, J., Abed, F., Selmi, M. A., Moran, J., Colado, J. C., \& Rebai, H. H. (2021). An eightweeks resistance training programme with elastic band increases some performance-related parameters in pubertal male volleyball players. Biology of Sport, 39(1), 
219-226. DOI: https://doi.org/10.5114/ biolsport.2021.101601.

Kaster,T., Dooley, F. L., Fitzgerald, J. S., Walch,T. J., Annandale, M., Ferrar, K., Lang, J. J., Smith, J. J., \&Tomkinson, G. R. (2020). Temporal trends in the sit-ups performance of 9,939,289 children and adolescents between 1964 and 2017. Journal of sports sciences, 38(16), 1913-1923. DOI: https: / / doi.org/10.1080/02640414.2020.1763764.

Kmet, L. M., Cook, L. S., \& Lee, R. C. (2004). Standard quality assessment criteria for evaluating primary research papers from a variety of fields. DOI: https://doi.org/ 10.7939/R37M04F16.

Kordi, H., Sohrabi, M., Saberi,A., \& Attarzadeh, S. R. (2016). The effect of strength training based on process approach intervention on balance of children with developmental coordination disorder. Arch Argent Pediatr, 114(6), 526533. DOI: https://doi.org/10.5546/aap.2016.eng.526.

Kovacevic, A., Mavros, Y., Heisz, J. J., \& Singh, M.A. F. (2018). The effect of resistance exercise on sleep: a systematic review of randomized controlled trials. Sleep medicine reviews, 39, 52-68. DOI: https://doi.org/10.1016/ j.smrv.2017.07.002.

Le-Cerf, L., Valdés-Badilla, P., \& Guzmán, E. (2022). Efectos del entrenamiento de fuerza sobre la condición física en niños y niñas con sobrepeso y obesidad: una revisión sistemática. Retos, 43, 233-242.

Lloyd, R. S., Faigenbaum, A. D., Stone, M. H., Oliver, J. L., Jeffreys, I., Moody, J. A., Brewer, C., Pierce, K. C., McCambrigde, T. M., Howard, R., Herrington, L., Hainline, B., Micheli, L. J., Jaques, R., Kraemer, W. J., McBride, M.G., Best, T. M., Ramirez, R., Chu, D. A., ... Myer, G. D. (2014). Posicionamiento sobre el entrenamiento de fuerza en jóvenes. Consenso Internacional de 2014. Revista de la Federación Española de Medicina del Deporte y de la Confederación Iberoamericana de Medicina del Deporte, 31(2), 111-124.

López, M. R., Cerda, F. J., Oñate, C. J., \& Lagos, R. (2021). Estado nutricional y fuerza de tren inferior: diferencias entre sexo y área geográfica entre niños y niñas. Retos, 42, 612-617. DOI:10.47197/retos.v42i0.88049

Lubans, D. R., Sheaman, C., \& Callister, R. (2010). Exercise adherence and intervention effects of two school-based resistance training programs for adolescents. Preventive Medicine, 50(1-2), 56-62. DOI: https://doi.org/ 10.1016/j.ypmed.2009.12.003.

Martínez-Pérez, P., \&Vaquero-Cristóbal, R. (2021). Revisión sistemática del entrenamiento de fuerza en futbolistas preadolescentes y adolescentes. Retos, (41), 272-284. DOI: https: / / doi.org/10.47197/retos.v0i41.82487.

Miranda-Aguilar, D., Valdés-Badilla, P., Herrera-Valenzuela, T., Guzmán-Muñoz, E., Branco, B. H. M., MéndezRebolledo, G., \& López-Fuenzalida, A. (2020). ¿ Bandas elásticas o equipos de gimnasio para el entrenamiento de adultos mayores? Retos: nuevas tendencias en educación físi- ca, deporte y recreación, 37, 370-378.

Moher, D., Shamseer, L., Clarke, M., Ghersi, D., Liberati, A., Petticrew, M., Shekelles, P., Stewart, L.A., \& PRISMA Group. (2015). Preferred reporting items for systematic review and meta-analysis protocols (PRISMA-P) 2015 statement. Systematic Reviews,4(1). DOI: https:// doi.org/10.1186/2046-4053-4-1.

Moliner-Urdiales, D., Ruiz, J. R., Ortega, F. B., Jiménez-Pavón, D., Vicente-Rodriguez, G., Rey-López, J. P., MartínezGómez, D., Casajús, J. A., Mesana, M. I., Marcos, A., Noriega-Borge, M. J., Sjöström, M., Castillo, M. J., \& Moreno, L.A. (2010). Secular trends in health-related physical fitness in Spanish adolescents: the AVENA and HELENA studies. Journal of Science and Medicine in Sport, 13(6), 584-588. DOI: https://doi.org/10.1016/ j.jsams.2010.03.004.

Özsu, Ý. (2018). Effects of 6-Week Resistance Elastic Band Exercise on Functional Performances of 8-9 Year-Old Children. Journal of Education and Training Studies, 6(12a), 23-28. DOI: https://doi.org/10.11114/ jets.v6i12a.3887.

Pochetti, J., Ponczosznik, D., \& Rojas, P. (2018). Entrenamiento de la fuerza en niños y adolescentes: beneficios, riesgos y recomendaciones. Archivos Argentinos Pediatría, 116(6), 82-91. DOI: https://doi.org/10.5546/ aap.2018.s82.

Riel, H., Matthews, M., Vicenzino, B., Bandholm,T., Thorborg, K., \& Rathleff, M. S. (2016). Efficacy of live feedback to improve objectively monitored compliance to prescribed, home-based, exercise therapy-dosage in 15 to 19 year old adolescents with patellofemoral pain-a study protocol of a randomized controlled superiority trial. $B M C$ musculoskeletal disorders, 17(1), 1-12. DOI: https:// doi.org/10.1186/s12891-016-1103-y.

a ahin, G., Aslan, M., \& Demir, E. (2016). Short-term effect of back squat with an elastic band on the squat and vertical jump performance in trained children. Journal of Physical Education and Sport, 16(1), 97. DOI: https://doi.org/ 10.7752/jpes.2016.01016.

Santos, J., Flauzino, A., Kirsch, J., Castilho, A., Priscila, A, \& Marcelo, C. (2019). Effects of training with elastic resistance versus conventional resistance on muscular strength: A systematic review and meta-analysis. SAGE Open Medicine. DOI: https://doi.org/10.1177/ 2050312119831116.

Terraza-Rebollo, M.; Baiget, E.; Corbi, F. \& Planas, A. (2017). Efectos del entrenamiento de fuerza en la velocidad de golpeo en tenistas jóvenes. Revista Internacional de Medicina y Ciencias de la Actividad Física y el Deporte, 17 (66) pp. 349-366. DOI: https://doi.org/10.15366/ rimcafd2017.66.009.

Urrútia, G., \& Bonfill, X. (2010). PRISMA declaration: a proposal to improve the publication of systematic reviews and meta-analyses. Medicina Clínica, 135(11), 507-511. DOI: https://doi.org/10.1016/j.medcli.2010.01.015. 